\title{
Social Studies: The Plan for New Zealand's Schools
}

\author{
Brian Crittenden
}

I

N 1993, the New Zealand Ministry of Education adopted a 'curriculum framework' of seven key learning areas for primary and secondary schools. These are: Science, Mathematics, Languages, Social Sciences, Technology, Health and Physical Wellbeing, the Arts. Documents setting out the essential learning in each area have been in preparation since that time. This discussion focuses on the 'core statement' for the Social Sceinces learning area, with special reference to criticisms raised by the Auckland-based Education Forum.

The first draft of the curriculum for Social Studies was published in 1994. After considerable debate, much of it through the national media, a revised draft was issued in 1996. The statement appeared in its final form in 1997 under the title Social Studies in the New Zealand Curriculum.

The Executive Committee of the Education Forum has about a dozen members (several from the manufacturing, commercial, and agricultural sectors along with a number of principals of private and state schools). The Forum contributes to debate on educational policy in New Zealand, and sponsors publications for this purpose. In relation to the Social Studies curriculum, it published a submission on the first and revised drafts (Education Forum, 1995, 1996), and issued a short media statement on the final version. Dr Geoffrey Partington contributed substantially to the two submissions. Professor Kenneth Minogue wrote a Foreword to the second.

In the context of the 'curriculum framework', Social Studies is interpreted as an integrated approach to the social sciences and aspects of the humanities. Its main stated aim is to 'enable students to participate in a changing society as informed, confident, and responsible citizens' (Ministry of Education, 1997:8). To achieve this aim, the Social Studies curriculum is designed to develop a broad understanding of people in the New Zealand society and in the wider contexts of its place in the Pacific, Asia, and the world generally.

\section{Comparisons with Recent Australian Curriculum Planning}

Before turning to the Education Forum's assessment of the Social Studies program, it may be of interest to note some comparisons with recent developments in programs for schooling in Australia. At its meeting in Hobart in 1989, the Australian Education Council (AEC), made up of State ministers for education and the Com- 
monwealth minister, issued a statement on agreed national goals for schooling. This was the beginning of the process for the development of 'national curriculum profiles' in eight 'key learning' areas (Art, English, Health and Physical Education, Languages other than English, Mathematics, Science, Studies of Society and Environment, Technology). With some minor variations in nomenclature, the later New Zealand list is the same, except that it treats English and other languages as a single learning area.

Final draft 'profiles' were presented to the AEC meeting in 1993. At this meeting, it was decided that the profiles would be referred to States and Territories, which would take responsibility for adapting their content. In Victoria, for example, its 'Curriculum and Standards Framework' accepted the eight key learning areas agreed to nationally, each organised at seven levels of difficulty covering the compulsory years of schooling. The general curriculum statement for Studies of Society and Environment (SOSE) was published in 1995 (Board of Studies, 1995). Like the other learning areas, its main content and processes are set out in several 'strands', which are the same at each level. In SOSE, there are five strands: time, continuity and change (drawing on the discipline of history); place and space (geography); culture (anthropology, religion, sociology); resources (economics and related disciplines, geography, environmental studies); natural and social systems (economics, ecology, sociology). SOSE emphasises three main processes in each strand at every level: investigate, communicate, participate. (It should be noted that the second and third processes involve a good deal of overlap.)

An examination of the structure of Social Studies in the New Zealand Curriculum reveals that it too has five 'strands' with essentially the same titles and background disciplines as in SOSE. There are also three key processes: inquiry, value exploration, social decision-making. Although they include substantially the same activities as in the SOSE processes, the New Zealand program gives more emphasis to the examination and clarification of each individual's values and those of others as they concern social issues.

One important difference is that material in the Social Studies core statement is set out at eight levels and covers all the years of primary and secondary schooling. As well, it explicitly refers to five perspectives: bicultural (Maori and European), multicultural, gender, current affairs, the future. As we shall see, the distinction between the first two raises difficulties. (The curriculum material, it should be noted, is published in both Maori and English.) With the exception of the first, these perspectives are, in fact, included in SOSE. Under the multicultural perspective, explicit attention is drawn to Aboriginal beliefs and practices.

While there are many variations of detail between the broad curriculum outlines presented in SOSE and the New Zealand statement, they are substantially similar in objectives and design. Perhaps the main differences are, first, that SOSE puts a little more emphasis on an understanding of the disciplines that relate to the strands, while the New Zealand program gives somewhat more attention to learning to participate as a responsible member of society; and second, that the study of values has a more central place in the Social Studies statement than in that of SOSE. 
Despite the striking similarities between the New Zealand curriculum framework and the common learning areas for Australian schools (devised somewhat earlier) and, with particular bearing on the present topic, between the general program for Social Studies in New Zealand and for SOSE in Australia (again, produced somewhat earlier), it is surprising that the former makes no reference to the latter. I should note that detailed course advice for teachers has now been produced (at least in Victoria) for all strands of SOSE at every level. $\quad$ While something similar will probably be prepared in New Zealand, the discussion here refers to the Social Studies 'core statement'.

\section{Criticisms by the Education Forum}

The two submissions by the Education Forum on the draft Social Studies program (and the media release on its final form) make the same main criticisms. It seems that the designers of the program were largely unmoved by the Education Forum's arguments.

The first draft. In reference to the first draft, the key deficiencies, in the Forum's view, can be summarised as follows.

First, there is an exaggerated emphasis on the learning of skills at the expense of content, and a distorted interpretation of what counts as a skill. Learning to communicate clearly, understanding the causes of a significant historical event, applying statistical methods appropriately in social scientific inquiry are all labelled, without qualification, as skills. However, such an activity as conjecturing and testing hypotheses in scientific inquiry cannot be treated as a skill in the same sense as, for example, being able to type.

Second, the felt needs and interests of learners play too dominant a role. The consequences are an overemphasis on the 'here and now', inadequate attention to expanding the experience and understanding of students, turning the teacher's work to that of a minder or coordinator rather than an active educator.

Third, in its approach to Maori and European society and culture, the curriculum statement concentrates on the positive features of the former and puts distorted emphasis on the negative features of the latter. In addition, it underplays the influence of the cultural traditions of the British Isles in New Zealand's development.

Fourth, the various strands fail to present the main content of the related intellectual disciplines in any systematic way. Despite the emphasis on skills, those that are distinctive of these disciplines are treated very inadequately.

The Forum concludes its first submission with serious doubts about the possibility of a social studies area in which the social sciences (and relevant disciplines from the humanities) can be integrated with intellectual coherence, in a way that is man-

\footnotetext{
${ }^{1}$ Although I would criticise many aspects of the general SOSE curriculum design, I have been a member of an 'expert panel' of four that has commented on the detailed course advice drafts. Their development has involved the contributions of a large number of individuals, schools and other organisations. The substantial course advice material helps to offset weaknesses in the general curriculum.
} 
ageable for teaching, learning and assessment over the course of schooling, and that can resist ideological distortion. It would prefer to see the social science disciplines established in their own right in the curriculum. It suggests that, if the attempt at an integrated social studies is persisted with, at least the study of geography should have a distinct place in the curriculum.

Some of the defects that the Forum submission identifies in the draft Social Studies statement reflect its conformity to principles of the general New Zealand Curriculum Framework, while others are deviations from the Framework. An example of the former is the exaggerated emphasis on skills. The second is illustrated in the deficient induction that the Social Studies program provides into history, geography, and economics.

The Forum submission draws attention to the defective treatment of key concepts. For example, there is no indication that there are serious disagreements over the notion of human rights, or that agreement at a general level does not exclude differences of interpretation in particular circumstances. Another example is the treatment of values. Emphasis on procedures for clarifying what values one actually holds and how one regards their relative importance is combined with the view that there are certain values that everyone should or should not hold. Again, in endorsing a general value (such as social justice), the curriculum statement does not take account of reasonable differences over its interpretation as a general value or, when there is agreement at this level, what counts as social justice in particular circumstances.

On the five 'strands', the Forum submission argues that they do not provide an effective organising structure for the learning of systematic knowledge and related skills. For example, 'Time, Continuity and Change' (drawing on history) consists largely of superficial activities and a random selection of topics. There is no clear indication of how skills for historical inquiry are to be developed. It is also unlikely that students would gain any sense of chronology.

Probably, the Forum's most serious criticism of the Social Studies draft is its lack of any substantial basis for an integrated, coherent treatment of the social science disciplines on which it draws. One could add that the objective of a coherent program would need to include aspects of the humanities: not only history, which may be regarded as belonging to both the social sciences and humanities, but also something of social philosophy and ethics.

Although the Forum prefers the study of separate social sciences, it suggests a set of significant themes that would be more likely to achieve a coherent program than the five strands. Each theme (for example, human control over nature) would, unlike the strands, bring together contributions from history, sociology, anthropology, economics and geography.

I should note that, while this approach is a better way of coordinating the relevant disciplines, it still does not address the issue of how students come to an adequate understanding of the radical differences in methodology and objectives of the contributing disciplines. 
The revised draft. The revised draft of Social Studies as one of the essential learning areas in the New Zealand Curriculum Framework was published in 1996. The Education Forum published its submission on the revised draft later in that year. As none of its key criticisms of the first draft was taken up, it is not surprising that the second submission makes very much the same points as the first.

In the Foreword, Kenneth Minogue notes, in particular, the excessive preoccupation with skills and their detachment from the structure of systematic inquiry and knowledge; a distorted and thus patronising picture of a bicultural New Zealand drawing equally on Maori and European traditions; the failure to acknowledge the British Isles as the most significant source of the culture and institutions that characterise New Zealand. His conclusion is that 'this entire project is so flawed as to be impossible to salvage' (p. xii).

The summary of the Forum's view of the revised draft claims that, apart from a few minor concessions (such as a 'faint reflection' of New Zealand's British background), it has not been substantially changed. Its reference to 'bicultural' gives predominant emphasis to features of Maori culture, and its description of New Zealand as both bicultural and multicultural remains unexamined. Values continue to be treated as subjective feelings, attitudes and the like; it is the personal ordering of these that each individual needs to clarify. At the same time, the revised draft promotes its own preferred scheme of values. The most serious criticism is the claim that the proposed program fails to provide students with a sound basis for acquiring a systematic understanding of New Zealand and its place in the world from the perspectives of history and the social sciences (p.xiii).

These criticisms are developed in the course of the Forum's submission on the revised draft. The submission argues that there is no clear defence of what social studies is as a 'distinct' subject and what its relationships are to the social science disciplines on which it draws. It reflects two interpretations of social studies in recent decades: as promoting a reform agenda for society; as a set of learning experiences directly linked with students' experiences in everyday social life. Major concepts (such as 'democracy' and 'liberalism') are neglected. History tends to be treated as a generalising social science such as physical geography or theoretical economics, rather than as the interpretation of particular developments in human society and culture. Skills (even 'critical thinking' and others of a non-generic kind) are treated as though the content in which they are developed is purely incidental to their exercise. The design of the strands still reflects fundamental weaknesses such as lack of internal sequence; confusion and overlap among them; neglect of structured areas of knowledge and critical understanding of their key concepts; biased selection of material (especially the neglect of the British cultural influence).

The Forum repeats its suggestion that, if a 'mixed' approach is to be maintained, it would be preferable to combine the three strands dealing with social organisation, culture, continuity and change over time. The past could be treated chronologically, and contemporary events could be treated in relation to students' experience on an 'expanding circle' model. The other two strands would each be based directly on a social science (geography, economics). 
The Social Studies 'core statement' was published in its final form in October 1997. In January of the previous year the Ministry of Education had published an analysis of responses to the revised draft statement. The only significant difference in the final version is the identification (with some elaboration) of three basic processes: inquiry, value exploration, social decision making. No mention is made of them in the summary of responses. A table sets out, in a general way, how one or more of these processes relate to each of seven broad skills identified as essential to the objectives of social studies. (These skills were listed in each of the draft statements.)

The Education Forum returned to the fray in a media release dated 30 October 1997). It reiterates basic criticisms made of the two drafts. First, the nature of 'social studies' remains vague. There are no clear criteria for distinguishing signifcant from trivial learning in this area. Although there are many references to 'knowledge', the statement gives little indication of what the crucial content is. Second, the Social Studies curriculum is largely an instrument for advancing government policy on biculturalism in New Zealand. The British origins of the society continue to be underplayed, being treated in the broad category of European influence. Third, the integrated approach involves difficulties for the study of such subjects as history and geography, and offers no clear compensatory advantages over their treatment as separate disciplines.

The Forum's criticisms of the Social Studies core statement are, I believe, substantially correct. I would endorse, in particular, two of the major points. First, the principles of integration relating to the key contributing disciplines are weak. They fail to take adequate account of the distinctive nature of these disciplines and how this affects their study as part of general education. Second, there is an exaggerated emphasis on skills. It seems to be supposed that they are all generic attainments and can be acquired and exercised regardless of particular content. The statement identifies seven essential skills, each of which is related in overlapping ways to three processes. The achievement objectives and indicators at the eight levels for each of the five strands are set out mainly in terms of explaining, identifying, and describing (with reference to a wide range of loosely connected content). The whole document has a mechanical, artificial air about it, like a set of instructions for assembling and using an appliance.

The New Zealand document is, as I noted earlier, very similar to the general outline for SOSE in Australian schools. However, the latter (at least in the Victorian version) does emphasise that one of its goals is to develop students' understanding of concepts in the underlying disciplines. The detailed course advice for teachers that has been produced in Victoria does give more attention to the content of the contributing disciplines and its close relationship to the exercise of critical inquiry and other skills within these disciplines. If the equivalent of course advice material is developed in New Zealand, it may go some distance in meeting the criticisms of the Education Forum. 


\section{Social Sciences and their Study in the Curriculum}

The nature of the social sciences and a number of the humanities has been a topic of close attention in recent decades. During this time there has also been considerable debate on integrated school curricula in the social sciences and humanities, and a number of significant efforts at such curriculum design. ${ }^{2}$

In the 19th century, strong efforts were made to develop the study of social systems through disciplines that conformed to the nomothetic model of the natural sciences. Sociology (under Auguste Comte's influence) emerged as the clearest example. The character of others was more complex. Anthropology, for example, developed in the context of the colonial activities of European nations. For a long time, it relied on participant observation or a mainly idiographic model. It had links with oriental and classical studies. These were concerned mainly with the analysis of significant texts and were identified as studies in the humanities.

Anthropology, economics, geography, political science, psychology, and legal studies became well established in the early part of the 20th century as disciplines in universities. Although they aspired to the methodology of the natural sciences, they were linked by their objects of study to the humanities. At an organisational level, they came to be accepted as occupying a middle ground between the natural sciences and the humanities.

In recent decades the study of fields that involve the perspectives of several, if not all, the social sciences has substantially increased. In particular, these refer to occupations and to complex social issues and matters of public policy. The presence of ethical issues, the increased attention to historical background, and the growth of 'cultural studies' in the humanities have all contributed to a close relationship between the social sciences and the humanities. One of the most important consequences has been the development of interpretative and other qualitative methods in the social sciences.

This change has been encouraged by a modifying of the commitment to nomothetic methodology within the natural sciences themselves. But more important has been the much clearer recognition that the radical differences between social and physical facts call for corresponding differences in the purposes and methods of systematic inquiry. Because human beings are purposive agents, with a sense of self-identity shaped by engagement in institutions that reflect rules of human design, they are very different as objects of systematic study from those of the natural sciences. It is now more commonly recognised that moral and other values are part of the fabric of social scientific inquiry: not simply objects of such inquiry, but constituents of the process and calling for justification in their interpretation and relative weighting. The methodology of the social sciences includes normative and interpretative dimensions as well as the quantitative.

${ }^{2}$ Discussions of the underlying issues include Crittenden (1981:ch. 5); Eisner (1979); Hill (1994); Hirst (1974); Pring (1976); Walsh (1993:ch. 9); Warwick (1974). Examples of integrated curricular designs for social sciences, humanities include Bruner (1965) (and see Bruner, 1968, ch. 5); Stenhouse (1970); Keele Integrated Studies Team (1972); Schools Council Moral Education 8-13 Project (1978). 
Within the social sciences the relative emphasis on these dimensions of methodology varies. Sociology, economics and social psychology are examples of social sciences in which the quantitative has a substantial place. By contrast, the discipline of history, as Isaiah Berlin (1997) has clearly argued, is predominantly concerned with depicting the whole pattern of events, eras and so on, not with establishing general laws. It uses quantitative methods where appropriate, but is as much interested in the distinctive features of, say, a revolution as in those that reflect laws applicable to revolutions generally. Above all, historians must exercise sympathetic imagination in trying to understand the ways of thinking and so on of people in very different circumstances from their own. The soundness of such interpretation can be assessed. But the conclusions are not reached on the basis of either deductive or inductive argument. It is the particulars, not generalisations, that are the primary concern of historical study. The main categories with which the historian works are qualitative, such as plausibility, likelihood, and sense of reality. History reflects a general condition of systematic inquiry: the richer the content of a science, the less rigorous are its explanatory and predictive theories. The price that some social sciences pay for precise laws (in economics, for example) is inaccuracy in prediction when these laws are applied to the complex circumstances of social life.

There are difficulties in Berlin's account of incommensurable goods, and more can probably be done than he allows in identifying likely patterns for the future based on what has happened in the past. But he highlights the distinctive methodological features of historical inquiry. These place it firmly among the humanities as well as the social sciences, and illustrate how distorting it is to treat the social sciences as though they conformed to a single methodological paradigm.

In examining the nature of the disciplines, Stephen Toulmin has drawn attention to characteristics that have a crucial bearing on their treatment in the school curriculum. As he points out, disciplines are not static ahistorical constructions. They are 'historically evolving collective enterprises that are institutionally organised' (1972:148). There is a continual interaction of processes and content. Given this dynamic character, they are distinguished at any time by their set of common questions and problems and their specific common aims. In relation to the latter, he stresses differences in epistemological objectives: causal generalisations; making phenomena intelligible in terms of human reasons and motives; interpretations of meaning; evaluation; prescription. Disciplines differ according to their focus on one or a particular combination of such objectives. Depending on the range of objects being investigated and the objectives of inquiry, the concepts and theories of some disciplines ('compact') form a more logically coherent pattern than others ('diffuse'). According to Toulmin, some studies fall into the category of 'would-be' disciplines.

On the question of integrated studies, Toulmin notes that variations in the methods of inquiry place constraints on their use as a basis for integration. Any approach that treats inquiry as a kind of constant and neglects the content and historical institutional character of disciplines is as defective as the older practice of treating content as timeless truth. The appropriate criteria for rational inquiry de- 
pend on the substantive concepts and the questions into which they enter. This condition is crucial for any defensible form of integration.

As Toulmin (1972:364) emphasises, each discipline is itself an integrating system, and all draw, in various ways, on common-sense knowledge, which itself does not form a fully coherent logical pattern. Among the significant ways in which disciplines can be integrated is in their contribution to the study of complex social issues which go beyond the scope of strictly disciplinable inquiry (for example, poverty, international peace, the treatment of the physical environment). Another basis for integration is provided by complex human activities such as government, the economic order, and education. If the objective is to reach a normative decision on macro-issues of public policy, social morality and so on, the application of a range of disciplines can provide illumination, but not a resolution.

It follows from Toulmin's account of disciplines that any satisfactory curriculum design needs to trace the paths that run from everyday knowledge to disciplined inquiry into a defined range of related topics, and then to the complex human questions that involve a combination of everyday knowledge and the systematic knowledge and ways of inquiring of relevant disciplines. In the development of multidisciplinary studies, the main warning implicit in Toulmin's analysis is against the superficiality of simply drawing particular bits and pieces from a number of disciplines, without any systematic study of their nature.

Unfortunately, this is the approach that both the SOSE framework and the New Zealand Social Studies core statement tend to take. In neither of them is there a systematic plan for developing, over the years of primary and secondary schooling, an understanding of the disciplines in the social sciences and humanities on which they draw: the key concepts, principles, and theories; the main purposes and methods of inquiry. Moreover, there are no significant integrating themes. With few exceptions, there is no interrelating, in either program, of what is to be learnt across the five discipline-based strands at each level.

The designers of the two programs do not seem to have given any serious attention to work that has been done in recent decades on integrated curricula in the social sciences and humanities (see footnote 2). One important example is Man: $A$ Course of Studies (MACOS), designed under Jerome Bruner's supervision in the mid-1960s, and reflecting strongly his theory of curriculum and psychology of learning. Many of the details of his theoretical position and of MACOS can be (and have been) criticised. What is important to note for our purpose, however, is the close attention given to the elements through which a significant integration of the contributing disciplines can be achieved. The program is designed to address, at various levels, three basic questions: What is human about human beings? How did they get that way? How can they be made more so? (Bruner slides over the problem of distinguishing desirable and undesirable features of being human.)

\footnotetext{
${ }^{3}$ have made some critical comments on Bruner in Crittenden (1979). Richard Jones (1972), who worked on the MACOS project, is critical of its neglect of the education of the emotions and the role they play in learning.
} 
Relevant disciplines are applied (with due attention to the structure of their content as well as methods of inquiry) to provoking questions about, and understanding of, five basic human activities: tool making, language, social organisation, the management of humans' extended childhood, and the human urge to explain the world.

MACOS was intended for students in the middle years of schooling. However, we know from Bruner's general theory of education that the 'structure' of the disciplines - that is, their basic concepts, theories, and methods of inquiry together with the pattern of relationship among the key elements - should be presented in ways that are appropriate for the dominant mode of learning at each main stage of development. Each discipline, he stresses, is a complex integrated unit in the whole curriculum. For Bruner, the emphasis moves from learning mainly through action ('enactive mode') to perceptual organisation and imagery ('iconic mode') to direct attention to symbols ('symbolic mode'). As children develop, it is a matter of each later mode becoming dominant, not of its displacing the earlier ones. Thus, for Bruner, the overall pattern of the curriculum should be a spiral one in which the same basic concepts, theories and skills are presented in accord with each dominant mode of representation. There are other ingredients in his proposal for a spiral curriculum - and, of course, it is not immune to criticism. The point to be emphasised here is that neither of the social studies curricula under discussion gives evidence that it is based on any clearly articulated theory of intellectual (and emotional) development through the years of formal schooling.

\section{Concluding Comments}

It will be obvious that, for the most part, I agree with the Education Forum's criticisms of the core statement on Social Studies for New Zealand schools, which also apply to the very similar SOSE frameworks in Australia. Some of the concerns may be met in the development of detailed advice for teachers (as for SOSE in Victoria). But the basic defects in the curriculum remain. Perhaps the best arrangement is to have a systematic study of history, geography and one or two other social sciences as distinct fields of knowledge and inquiry, and to include at various levels in the course of schooling a program designed in relation to a set of significant social issues. The latter would bring together content and methods from a range of social sciences - and also several of the humanities. In addition to history which, in various ways, belongs to both areas, these would certainly need to include elements of ethics and social philosophy. There would also be scope for drawing on literature and the arts.

Among the advantages of some such arrangement, at least two should be stressed. First, it enables students to focus on the distinctive content and methods in each of a balanced range of systematic intellectual disciplines; second, it provides the opportunity for students to learn how to apply these disciplines to significant

\footnotetext{
${ }^{4}$ The Humanities Project (Stenhouse, 1970) relates subjects in the humanities (and social sciences) to the discussion of controversial issues in complex areas of human experience, including war, education, the family, poverty, and race relations.
} 
issues whose complexity transcends the boundaries of any individual discipline in order to gain understanding, make informed decisions, and so on. What needs to be stressed is that no integrated curriculum will be educationally effective unless it respects the nature of the disciplines involved (both their central content and methods); provides significant topics that require the application of a range of disciplines; and matches the learning objectives to the students' general level of intellectual and emotional development and previous formal educational experience.

\section{References}

Berlin, I. (1997), 'The Concept of Scientific History', pp. $17-58$ in H. Hardy \& R. Hansheer (eds), The Proper Study of Mankind, Chatto \& Windus, London.

Board of Studies, Victoria (1995), Curriculum and Standards Framework: Studics of Socicty and Environment, Melbourne.

Bruner, J. (director) (1965), Man: A Course of Studies, Education Development Center, Boston.

Bruner, J. (1968), Towards a Theory of Instruction, Norton, New York.

Crittenden, B. (1979), 'Jerome Bruncr: Structure in Knowledge and Learning as the Key to Education', pp. 27-47 in J. D'Cruz and W. Hannah (eds), Perceptions of Excellence, The Polding Press, Melbourne.

(1981), Education for Rational Understanding, Australian Council for Educational Research, Melbourne.

Education Forum (1995), Social Studies in the New Zealand Curnculum: A Submission on the Draft, Astra DPS, Wellington (August).

- (1996), Social Studies in the New Zealand Curriculum: A Submission on the Revised Draft, Astra DPS, Wellington (October).

Eisner, E. (1979), The Educational Imagination: On the Design and Evaluation of School Programs, Macmillan, New York.

Hill, B. (1994), Teaching Secondary Social Studies in a Multicultural Society, Longman Cheshire, Melbourne.

Hirst, P. (1974), Knowledge and the Curriculum, Routledge \& Kegan Paul, London.

Jones, R. (1972), Fantasy and Feeling in Education, Penguin, Harmondsworth.

Kecle Integrated Studies Team (1972), Exploration Man: An Introduction to Integrated Studies, Oxford University Press, Oxford.

Ministry of Education (1997), Social Studies in the New Zealand Curriculum, Learning Media Ltd, Wellington.

Pring, R. (1976), Knowledge and Schooling, Macmillan, Melbourne.

Schools Council Moral Education 8-13 Project (1978), Startine, Longman, London. 


\section{Brian Crittenden}

Stenhouse, L. (director) (1970), The Humanities Project, Schools Council Publications, London.

Toulmin, S. (1972), Human Understanding, Vol 1, Clarendon Press, Oxford.

Walsh, P. (1993), Education and Meaning, Cassell, London.

Warwick, D. (ed.) (1974), Integrated Studies in the Secondary School, University of London Press, London. 